\title{
RESULTADOS OBTIDOS COM A IMPLANTAÇÃO DO ENTERPRISE RESOURCE PLANNING EM UMA INDÚSTRIA METALÚRGICA: SOB A PERSPECTIVA DOS COLABORADORES
}

\author{
RESULTS OBTAINED WITH THE IMPLEMENTATION OF ENTERPRISE \\ RESOURCE PLANNING IN A METALLURGICAL INDUSTRY: FROM THE \\ PERSPECTIVE OF EMPLOYEES
}

Renan Borges Borghi Especialista em Gestão de Logística e Gestão Empresarial e Engenheiro de Produção. Universidade do Oeste Paulista - UNOESTE. Presidente Prudente, São Paulo - Brasil.

borghi_14@hotmail.com

Gustavo Yuho Endo Mestre em Administração e Doutorando em Engenharia de Produção. Business School Unoeste - UNOESTE. Presidente Prudente, São Paulo - Brasil. gustavo_endo@yahoo.com.br

Eric Vinicius Lucion Mestre em Administração. Univel Centro Universitário - UNIVEL. Cascavel, Paraná - Brasil eric.lucion@univel.br

\author{
Ronaldo Jose Seramim \\ Mestre em Administração. \\ Universidade Federal da Fronteira Sul - UFFS. \\ Laranjeiras do Sul, Paraná - Brasil. \\ ronaldoseramim@yahoo.com.br
}

Resumo: O objetivo deste artigo foi descrever e compreender as experiências dos colaboradores das áreas operacionais e estratégicas após a implantação do sistema Enterprise Resource Planning (ERP), a partir do estudo de caso de uma indústria metalúrgica fabricante de equipamentos para a avicultura, no centro-oeste paulista. Trata-se de um estudo de caso com análise qualitativa a partir de entrevistas semiestruturadas, realizadas com seis colaboradores envolvidos na implantação do ERP. Os principais achados da pesquisa estão relacionados com as melhorias significativas no fluxo dos processos, níveis de controle, integração de dados, registros de movimentações, facilidade de acesso à informação e melhorias no clima organizacional, dificuldades de adaptação ao novo sistema remanescente, falta qualidade nas informações e padrões nos procedimentos. Os colaboradores sugerem necessidade de treinamentos para uma melhor experiência com o ERP. Conclui-se que a comunidade interessada na implantação do ERP pode se atentar às dificuldades que se dão no momento da implantação.

Palavras-chave: Gestão empresarial. ERP. Indústria. Colaboradores.

Abstract: The aim of this article was to describe and understand the experiences of employees in the operational and strategic areas after the implementation of the Enterprise Resource Planning (ERP) system, based on the case study of a metallurgical industry that manufactures equipment for poultry, in the Midwest of São Paulo. This is a case study with qualitative analysis based on semi-structured interviews, carried out with six employees involved in the implementation of the ERP. The main findings of the research are related to significant improvements in the flow of processes, levels of control, data integration, movement records, ease of access to information and improvements in the organizational climate, there are still difficulties in adapting to the new system remaining, lack quality of information and standards in procedures. Employees suggest the need for training, for a better experience with ERP. It is concluded that the community interested in the implementation of ERP can be attentive to the difficulties that occur at the time of implementation.

Keywords: Business management. ERP. Industry. Collaborators.

Cite como

American Psychological Association (APA)

Borghi, R. B., Endo, G. Y., Lucion, E. V., \& Seramim, R. J. (2021, jan./jun.). Resultados obtidos com a implantação do enterprise resource planning em uma indústria metalúrgica: sob a perspectiva dos colaboradores. Revista Inovação, Projetos e Tecnologias - IPTEC, São Paulo, 9(1), 90-108. https://doi.org/10.5585/iptec.v9i1.19064. 


\section{Introdução}

Em um mercado globalizado, os sistemas de informação permitem que as empresas sejam mais competitivas e dinâmicas diante dos seus concorrentes (Silvério, Santos, \& Bastos, 2019). Dessa forma, as empresas são induzidas ou obrigadas a se reinventar a cada dia, para quebrar seus paradigmas, modificando fluxos e procedimentos em busca de melhores práticas organizacionais (Nguyen et al. 2021;Almeida, Kudlawicz-Franco, \& Sokulski, 2016).

O uso das tecnologias de informação, em específico os softwares de gestão empresarial conhecidos como Enterprise Resource Planning (ERP), aumentou a partir de 1990 nos Estados Unidos e Europa e, no Brasil, cresceu a partir de 1996. Porém, ao longo do tempo, notou-se que os resultados dos sistemas ERP não dependem exclusivamente de hardware e software (Jesus \& Oliveira, 2007; Souza \& Zwicker, 2003), pois existem barreiras ou dificuldades que as organizações poderão encontrar, desde falta de planejamento para implantação; possível falta de recursos financeiros; não estar preparada para realizar mudanças tecnológicas internas; barreiras na estrutura organizacional e dificuldades no relacionamento entre os colaboradores (Jesus \& Oliveira, 2007).

Apesar das barreiras e dificuldades, é evidente que, se bem aplicado, um sistema integrado de gestão torna o controle de estoque mais eficiente, propiciando auxílio à tomada de decisão; permitindo programação do controle logístico; gerando maior desempenho e controle de custos (Silvério et al., 2019), resultando em um processo de transformação organizacional (Gozzi, Fedichina, Olivo, \& Machado, 2006).

Portanto, as pesquisas sobre a implantação de sistemas ERP foram objeto de estudo de vários autores ao longo dos anos, como: Esteves, Carvalho e Santos (2000), Souza e Zwicker (2003), Gozzi et al. (2006), Jesus e Oliveira (2007), Peleias, Trevizoli, Cortes, \& Galegale (2009), Almeida et al. (2016), Costa et al. (2016), Giorgia e Santos (2015), Roselli e Cerqueira (2016); Ferreira, Penha \& Kniess (2019); Silvério et al. (2019) e Nguyen et al. (2021).

Nesse sentido, a literatura sugere novos estudos a serem realizados durante e após a implantação de um sistema ERP, a fim de avaliar as barreiras e dificuldades evidenciadas na teoria. Tais estudos se fazem necessários, uma vez que a implantação de ERP é vista como um processo complicado, que exigirá investimentos elevados e que pode impactar no funcionamento da organização e os resultados possam aparecer somente a médio e longo prazo (Dumitru \& Florescu, 2009).

Diante do contexto apresentado, é relevante compreender quais as percepções de mudanças observadas pelos colaboradores após a implantação do sistema de gestão integrado? 
Dessa forma, o objetivo foi descrever as experiências dos colaboradores das áreas operacionais e estratégicas após o processo de implantação de um sistema ERP, a partir do estudo de caso de uma indústria metalúrgica fabricante de equipamentos para a avicultura, situada no centrooeste paulista, classificada como de médio porte.

Para atendimento a este objetivo, a pesquisa se desenvolveu a partir da coleta de informações junto a seis entrevistados diretamente envolvidos com o processo de implantação do sistema na organização, buscando evidenciar suas experiências gerais relativas a seis categorias de análise encontradas no arcabouço teórico tomado como base de construção do estudo.

Indo além deste trecho introdutório, a pesquisa se desenvolve em outras quatro seções. Na seção seguinte, a fundamentação teórica traz o arcabouço de discussões práticas e literais sobre o tema abordado. Na subsequente, a metodologia de investigação e construção deste estudo é apresentada, seguida da apresentação e discussão dos resultados e, por fim, são apresentadas as considerações finais do estudo.

\section{Fundamentação teórica}

Nesta seção é apresentado o arcabouço teórico que se fez base de estruturação para este estudo, sendo dividido na apresentação do conceito de ERP e os diferentes espectros que implicam na implantação deste nas organizações.

\subsection{Enterprise Resource Planning (ERP)}

O conceito de sistema Enterprise Resource Planning (ERP) surgiu a partir da necessidade de um software que pudesse integrar todas as informações da organização para auxiliar os gestores no momento da tomada de decisão. O ERP é uma consequência da evolução computacional e econômica, que iniciou na década de 1970 com o MRP (Material Requirement Planning) ou planejamento dos recursos de manufatura (MRP II), ganhando muita força a partir da década de 1990 (Jesus \& Oliveira, 2007).

Os ERPs podem ser usados por empresas de vários segmentos e tamanhos, a sua utilização racional impõe mudanças nos processos administrativos, forçando os profissionais a se aprimorarem (Peleias et al., 2009). Apesar da imposição de mudanças, o retorno sempre é o melhor e maior controle de atividades e/ou processos, integração com fornecedores, redução de custos e acesso à informação em tempo real (Giorgia \& Santos, 2015). Além disso, é uma forma de alcançar a integração da cadeia de suprimentos, que resulta em melhoria de produtividade e 
obtenção de vantagens competitivas (Gomes, 2013). Desta forma, a implantação de um sistema ERP não pode ser traduzida ou pensada como uma simples instalação de um software, pois trata-se de uma ferramenta estratégica (Souza \& Zwicker, 2003).

\subsubsection{Relação entre ERP e planejamento estratégico organizacional}

A relação entre ERP e o planejamento é discutida por vários pesquisadores, apontando para resultados que corroboram a sua importância (Gomes, 2013; Gozzi et al., 2006; Oliveira \& Hatakeyama, 2012) e necessidade de ocorrência (Pinto, Ramirez, \& Grandón, 2017), assim como para as limitações encontradas nos sistemas em fornecer alinhamento aos objetivos de longo prazo das organizações (Ferreira \& Kuniyoshi, 2015; Gomes, 2013).

É evidenciado a partir das experiências e pesquisas realizadas que o planejamento de ERP deve ser condizente com a estrutura organizacional existente, a qual avalia-se a cultura presente e a capacidade dos colaboradores no possível manejo do sistema (Ferreira \& Kuniyoshi, 2015; Giorgia \& Santos, 2015). A definição de metas e objetivos sobre o sistema também se faz de importante ocorrência (Souza \& Zwicker, 2000).

Ainda, considerando a integração promovida pelo sistema nos diversos setores organizacionais e logo, o envolvimento de grande parte dos colaboradores, a comunicação clara da alta direção aos subordinados sobre a decisão de implantar o ERP e as mudanças previstas no processo são fundamentais (Ferreira \& Kuniyoshi, 2015; Gozzi et al., 2006; Roselli \& Cerqueira, 2016).

Além do manejo com os colaboradores, a escolha da ferramenta a ser utilizada deve ser analisada exaustivamente (Giorgia \& Santos, 2015; Gomes, 2013), a fim de atender adequadamente aos objetivos organizacionais e fornecer alinhamento aos planos de longo prazo do negócio (Grandón, Ramírez-Correa, \& Rojas, 2018; Pinto et al., 2017).

Como resultado, o planejamento bem realizado e comunicado proporciona economia de custos gerais de implantação (Gomes, 2013), menor resistência e necessidade de treinamento (Gozzi et al., 2006), aumento de controle a partir dos relatórios gerados compatíveis com o negócio, apoio às estratégias, aumento de competitividade (Oliveira \& Hatakeyama, 2012; Roselli \& Cerqueira, 2016) e da capacidade adaptativa da organização (Groenewald \& Okanga, 2019; Oliveira \& Hatakeyama, 2012). 


\subsubsection{Comprometimento da alta direção sobre o processo de implantação}

O planejamento ocorre em prática pela alta gerência da organização, sendo o seu sucesso condicionado ao comprometimento dos componentes decisórios da empresa na comunicação transparente com os colaboradores, na reunião eficiente dos recursos organizacionais (Ferreira \& Kuniyoshi, 2015; Grandón et al., 2018; Pinto et al., 2017; Roselli \& Cerqueira, 2016; Souza \& Zwicker, 2000) e na gestão das mudanças provocadas pelo sistema (Gomes, 2013; Macêdo, Gaete, \& Joia, 2014; Pavón González, Puente Baró, Infante Abreu, \& Blanco González, 2018; Souza \& Zwicker, 2000).

Dada a circunstância da modificação de um modelo departamentalizado para um processo integrado de gestão (Ferreira \& Kuniyoshi, 2015; Souza \& Zwicker, 2000), o qual impactará nas relações de poder e estrutura organizacional (Macêdo et al., 2014), exigindo dos gestores capacidade de gestão de equipes multidisciplinares, integrando objetivos organizacionais aos dos colaboradores (Ferreira \& Kuniyoshi, 2015; Roselli \& Cerqueira, 2016).

Além da modificação nas relações e integração da empresa, o processo tende a valorar a ocorrência de resistência e conflitos entre gerentes e colaboradores (Macêdo et al., 2014) e também entre setores (Ferreira \& Kuniyoshi, 2015; Souza \& Zwicker, 2000), além de ser possível o surgimento de aversão ao sistema e boicote do mesmo (Gozzi et al., 2006; Giorgia \& Santos, 2015), na qual o comprometimento da gestão é mais uma vez requisitado (Gomes, 2013; Souza \& Zwicker, 2000).

Assim, o gestor é o guia do processo e responsável pelo projeto (Pinto et al., 2017), tanto alinhando os objetivos do plano como servindo de modelo aos colaboradores no processo de implantação (Ferreira \& Kuniyoshi, 2015), devendo possuir conhecimento singular sobre a organização, plano e sistema a ser implantado, assim como capacidades gerenciais significativas (Gomes, 2013; Pavón González et al., 2018).

Enquanto níveis estratégicos se dedicam a melhor escolha do sistema de acordo com seus objetivos e alinhamento organizacionais (Giorgia \& Santos, 2015; Gomes, 2013), os níveis táticos desenvolvem a comunicação e o suporte ao processo ao nível operacional (Ferreira \& Kuniyoshi, 2015; Gozzi et al., 2006), principal grupo de usuários diretos do sistema. 


\subsubsection{Percepção dos colaboradores sobre a implantação do sistema}

Vários estudos buscam entender a percepção dos diversos colaboradores sobre a implantação e utilização do sistema (Jesus \& Oliveira, 2007; Peleias et al., 2009), com o objetivo de direcionar ações que possibilitem melhoria no desempenho geral da ferramenta e utilização eficiente do grupo (Almeida et al., 2016; Macêdo et al., 2014; Souza \& Zwicker, 2000), uma vez que a adaptação inadequada dos usuários pode comprometer o projeto (Giorgia \& Santos, 2015).

Dentre os principais aspectos observados pelos autores está a resistência à mudança muitas vezes natural do indivíduo (Jesus \& Oliveira, 2007; Macêdo et al., 2014; Souza \& Zwicker, 2000), ou proporcionada por sistemas pouco funcionais (Macêdo et al., 2014), por falta de treinamento (Giorgia \& Santos, 2015), tempo hábil para adaptação (Almeida et al., 2016), comunicação adequada por parte da gestão (Ferreira \& Kuniyoshi, 2015), ou ainda por receio a manutenção dos postos de trabalho (Jesus \& Oliveira, 2007).

Ainda é possível observar por meio dos relatos que existe tendência ao aumento de responsabilidade, o que se não for corretamente trabalhado também impactará na resistência dos colaboradores ao sistema (Macêdo et al., 2014). Também existem relatos sobre a necessidade de angariar novos conhecimentos por parte dos colaboradores, o que é visto em pesquisas como algo positivo (Almeida et al., 2016; Peleias et al., 2009) e em outras como negativos (Jesus \& Oliveira, 2007).

\subsubsection{Treinamento dos colaboradores para o uso do ERP}

O treinamento dos colaboradores é algo bastante discutido na literatura, em que aborda a implantação de sistemas (Almeida et al., 2016; Gozzi et al., 2006; Grandón et al., 2018; Jesus \& Oliveira, 2007; Macêdo et al., 2014; Pinto et al., 2017) visto como a etapa de elevados custos dentro do processo (Esteves et al., 2000), e que dada a necessidade de angariar novos conhecimentos e as novas responsabilidades (Jesus \& Oliveira, 2007; Souza \& Zwicker, 2000) exigidas aos usuários finais é essencial para aumentar as chances de sucesso do mesmo (Ferreira \& Kuniyoshi, 2015; Giorgia \& Santos, 2015).

Além de reduzir as incertezas e inseguranças dos usuários sobre a utilização da nova tecnologia (Esteves et al., 2000; Oliveira \& Hatakeyama, 2012), o treinamento apresenta grande importância no fator redução de erros (Gozzi et al., 2006). Este ponto é significativo ao passo que erros em uma estrutura integrada interferem imediatamente nos resultados dos demais colaboradores e, logo, na estrutura como um todo (Jesus \& Oliveira, 2007). 
O treinamento prévio e bem estruturado, que não se limita à questões básicas de interface, mas com principal ênfase na entrada dos dados e nas responsabilidades de cada operador (Jesus \& Oliveira, 2007; Oliveira \& Hatakeyama, 2012; Souza \& Zwicker, 2000), otimiza o processo de implantação, além de reduzir custos futuros com erros e contratação de consultorias (Esteves et al., 2000; Gozzi et al., 2006), melhorando a adaptação do usuário ao novo modelo adotado (Giorgia \& Santos, 2015).

\subsubsection{Existência e comprometimento da área de TI}

Outro fator citado na literatura que potencializa o desempenho de implantação do ERP é a existência (Grandón et al., 2018; Peleias et al., 2009; Pinto et al., 2017) e comprometimento do setor de tecnologia da informação - TI (Ferreira \& Kuniyoshi, 2015; Macêdo et al., 2014) nas organizações.

Profissionais qualificados e com conhecimento em tecnologias se tornam fator importante para o suporte à implementação do sistema (Ferreira \& Kuniyoshi, 2015; Grandón et al., 2018; Macêdo et al., 2014; Pinto et al., 2017), e quando integrantes da equipe organizacional apresentam respostas mais rápidas aos problemas relativos à tecnologia e manuseio do sistema (Pavón González et al., 2018; Peleias et al., 2009; Silvério et al., 2019).

Contudo, é ressaltado que a existência de profissionais qualificados ou que dominam o software específico utilizado pela empresa é algo limitado no mercado (Correa \& Spinola, 2015). Ainda, os custos relativos à manutenção deste tipo de profissional no quadro de funcionários é elevada (Esteves et al., 2000).

A ausência do profissional de TI na organização amplia a necessidade de consultorias externas na implantação e adaptação da empresa com o sistema, sendo um dos fatores que contribuem para a aceleração do processo de implementação (Corrêa \& Spinola, 2015; Silvério et al., 2019; Souza \& Zwicker, 2003).

\section{$\underline{\text { 2.1.6 Necessidade de consultoria externa }}$}

A contratação de consultores para atuar no processo de escolha, implantação e adaptação é visto como algo importante em alguns estudos (Almeida et al., 2016; Esteves et al., 2000; Ferreira \& Kuniyoshi, 2015; Oliveira \& Hatakeyama, 2012; Souza \& Zwicker, 2003), pouco importante em outro (Pinto et al., 2017), e em um é até mesmo dificultoso (Correa \& Spinola, 2015). 
A partir da literatura entende-se que optar por consultoria externa possibilita a empresa agilidade nos processos e maior êxito no projeto (Ferreira \& Kuniyoshi, 2015; Souza \& Zwicker, 2003). O consultor pode agregar novos conhecimentos e metodologias, e deve ser capaz de apresentar planos de mudança de gestão do negócio (Esteves et al., 2000; Ferreira \& Kuniyoshi, 2015).

Contudo esta decisão deve considerar o cenário dos recursos internos da empresa, tanto no aspecto financeiro, como de flexibilidade da equipe em aceitar mudanças (Almeida et al., 2016; Esteves et al., 2000; Ferreira \& Kuniyoshi, 2015; Oliveira \& Hatakeyama, 2012; Pinto et al., 2017).

Além dos fatores internos, a escolha do consultor deve levar em consideração sua experiência sobre a ferramenta, visto que o grande número de softwares disponíveis no mercado acaba por elencar especificidades exigindo muitas vezes qualificação específica do consultor no ERP contratado (Correa \& Spinola, 2015; Ferreira \& Kuniyoshi, 2015).

\section{Metodologia}

Esta pesquisa é caracterizada como qualitativa e utilizou-se do estudo de caso, uma pesquisa empírica (Yin, 2018), com orientação epistemológica (paradigma) interpretativa, sendo que o objetivo principal é descrever e compreender as experiências dos colaboradores após o processo de implantação de um sistema ERP, ou seja, ex post facto (Appolinário, 2011; Bernardes, Muniz Junior, \& Nakano, 2019). O processo visa compreender como e porque por meio de diversas técnicas interpretativas as coisas acontecem, buscando alcançar o entendimento profundo do fenômeno (Cooper \& Schindler, 2016).

A coleta de dados ocorreu por meio de 06 entrevistas semiestruturadas, a qual possui perguntas já estabelecidas, com aprofundamento sobre algum tema (Appolinário, 2011), de 6 indivíduos de forma intencional, envolvidos com o processo de implantação do sistema ERP (Cooper \& Schindler, 2016) e, a observação participante direta, a qual um dos pesquisadores faz parte do quadro de colaboradores da empresa, esse envolvido diretamente no ambiente a qual a pesquisa foi realizada (Bernardes et al., 2019), assim foi possível contribuir na análise e discussões dos resultados. As questões estão descritas no Quadro 1 e foram preparadas a partir dos estudos de Almeida et al. (2016), Bancroft, Seip e Sprengel (1998), Caiçara Júnior (2012), Colangelo Filho (2001), Giorgia e Santos (2015), Sammon e Adam (2010) e Souza e Zwicker (2000). 
Os entrevistados pertenciam ao departamento do Planejamento, Programação e Controle da Produção (PPCP) e seus consecutivos líderes, escolhidos para possibilitar comparação entre a percepção da área operacional (2 pessoas) em relação à administrativa superior (4 pessoas).

Quadro 1 - Questões norteadoras da pesquisa

\begin{tabular}{|c|c|c|}
\hline Categoria & Questões & Autores \\
\hline $\begin{array}{l}\text { Planejamento } \\
\text { Estratégico }\end{array}$ & $\begin{array}{l}\text { 1) Como e em que momento (antes, durante ou após) você foi } \\
\text { informado sobre a implantação do ERP? 2) Em relação ao seu } \\
\text { departamento, quais fatores contribuíram para a implantação do ERP? } \\
\text { 3) Quais as dificuldades existentes após a implantação? 4) Quais fatores } \\
\text { contribuem para que a empresa não use plenamente o sistema ou da } \\
\text { melhor forma possível? }\end{array}$ & \multirow{6}{*}{$\begin{array}{l}\text { Almeida et al. } \\
\text { (2016); Giorgia e } \\
\text { Santos (2015); } \\
\text { Caiçara Júnior } \\
\text { (2012); Sammon } \\
\text { e Adam (2010); } \\
\text { Colangelo Filho } \\
\text { (2001); Souza e } \\
\text { Zwicker (2000); } \\
\text { Bancroft et al. } \\
\text { (1998). }\end{array}$} \\
\hline $\begin{array}{l}\text { Percepção do } \\
\text { Colaborador }\end{array}$ & $\begin{array}{l}\text { 5) Como eram os níveis de controle da empresa antes da implantação } \\
\text { do ERP? 6) Como ficaram os níveis de controle da empresa após a } \\
\text { implantação do ERP? 7) Quais mudanças você considera que foram } \\
\text { positivas? 8) Quais mudanças você considera que foram negativas? 9) } \\
\text { Você acredita que o ERP melhorou o fluxo de processos dentro do seu } \\
\text { departamento? Porquê? 10) Qual seu grau de satisfação com a } \\
\text { implantação do ERP? Avalie de } 1 \text { a } 10 \text { e justifique. }\end{array}$ & \\
\hline Treinamento & $\begin{array}{l}\text { 11) Como e em que momento (antes, durante ou após) você recebeu } \\
\text { treinamento para o uso do ERP? 12) Os treinamentos realizados com } \\
\text { você para o uso do ERP foram adequados? Avalie de } 1 \text { a } 10 \text { e justifique. } \\
\text { 13) Quais fatores devem ser analisados para melhorar o nível de } \\
\text { treinamento? }\end{array}$ & \\
\hline $\begin{array}{l}\text { Consultoria } \\
\text { Externa }\end{array}$ & $\begin{array}{l}\text { 14) Como a organização obteve ajuda de consultores externos para } \\
\text { implantação? } \\
\text { 15) Avalie de } 1 \text { a } 10 \text { e comente sobre a equipe de consultoria. }\end{array}$ & \\
\hline $\begin{array}{l}\text { Comprometimento } \\
\text { da Alta Direção }\end{array}$ & $\begin{array}{l}\text { 16) Como foi o envolvimento da alta administração no projeto de } \\
\text { implantação? } \\
\text { 17) Avalie de } 1 \text { a } 10 \text { o apoio da alta administração para realização dos } \\
\text { projetos e justifique. }\end{array}$ & \\
\hline $\begin{array}{l}\text { Comprometimento } \\
\text { da Área de TI }\end{array}$ & $\begin{array}{l}\text { 18) A infraestrutura oferecida atende o ERP? Justifique. 19) A equipe } \\
\text { de TI fornece apoio tecnológico para as necessidades do sistema e dos } \\
\text { processos? Avalie de } 1 \text { a } 10 \text { e justifique. }\end{array}$ & \\
\hline
\end{tabular}

Fonte: Elaborado pelos autores (2019).

O Quadro 2 apresenta o perfil dos trabalhadores e demais detalhes das entrevistas realizadas na fase de obtenção de dados. 
Quadro 2 - Perfil dos entrevistados

\begin{tabular}{|c|c|c|c|c|c|l|}
\hline Entrevistados & Departamento & Função & $\begin{array}{l}\text { Tempo de } \\
\text { Trabalho }\end{array}$ & Key-user & $\begin{array}{l}\text { Tempo de } \\
\text { Gravação }\end{array}$ & $\begin{array}{l}\text { Data da } \\
\text { Entrevista }\end{array}$ \\
\hline E01 & Operacional & $\begin{array}{c}\text { Apontador } \\
\text { de } \\
\text { Produção }\end{array}$ & 2 anos & Não & $\begin{array}{c}11 \text { minutos e } 45 \\
\text { segundos }\end{array}$ & $25 / 08 / 2019$ \\
\hline E02 & Operacional & $\begin{array}{c}\text { Apontador } \\
\text { de } \\
\text { Produção }\end{array}$ & 3 anos & Não & $\begin{array}{c}14 \text { minutos e } 8 \\
\text { segundos }\end{array}$ & 25/08/2019 \\
\hline E03 & Administrativo & $\begin{array}{c}\text { Analista de } \\
\text { PCP }\end{array}$ & 2 anos & Sim & $\begin{array}{c}16 \text { minutos e 1 } \\
\text { segundo }\end{array}$ & $22 / 08 / 2019$ \\
\hline E04 & Administrativo & $\begin{array}{c}\text { Programad } \\
\text { or de } \\
\text { Produção }\end{array}$ & 3 anos & Sim & $\begin{array}{c}8 \text { minutos e } 46 \\
\text { segundos }\end{array}$ & $26 / 08 / 2019$ \\
\hline E05 & Administrativo & $\begin{array}{c}\text { Programad } \\
\text { or de } \\
\text { Produção }\end{array}$ & 2 anos & Sim & $\begin{array}{c}6 \text { minutos e } 4 \\
\text { segundos }\end{array}$ & $25 / 08 / 2019$ \\
\hline E06 & Administrativo & $\begin{array}{c}\text { Assistente } \\
\text { de PCP }\end{array}$ & 8 anos & Não & $\begin{array}{c}7 \text { minutos e } 39 \\
\text { segundos }\end{array}$ & $25 / 08 / 2019$ \\
\hline
\end{tabular}

Fonte: Elaborado pelos autores (2019).

Os conteúdos produzidos foram organizados em 6 temáticas/categorias, permitindo aplicar a análise de conteúdo (Bardin, 2011). Em resumo, o percurso metodológico deste trabalho está resumido na figura 1.

Figura 1 - Percurso metodológico utilizado na pesquisa.

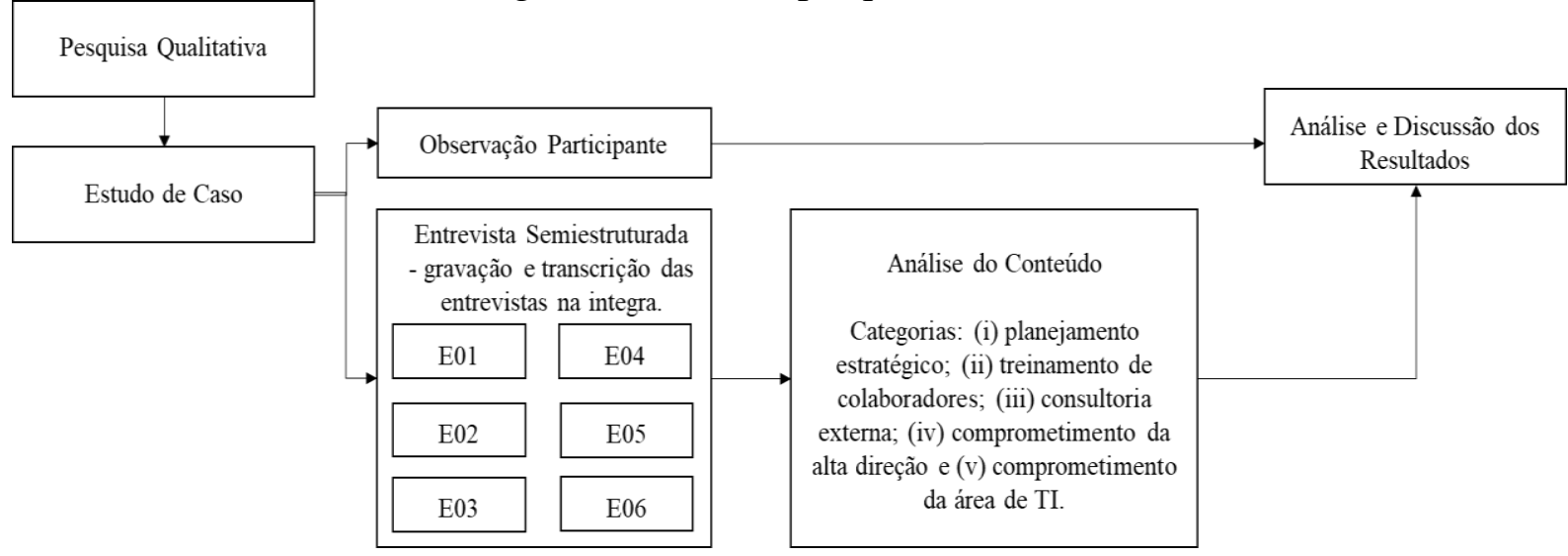

Fonte: Elaborado pelos autores (2019).

A empresa analisada neste estudo é uma indústria metalúrgica fabricante de equipamentos para a avicultura, seu portfólio de produtos é sistema vertical, sistema piramidal, fábrica de ração e peças de reposição e oferecem serviços de assistência técnica; atua no mercado desde a década de 1970; situada no centro-oeste paulista; de médio porte e emprega 350 colaboradores. 
Após as análises das entrevistas e das observações realizadas, foi possível identificar que depois da implantação do ERP tiveram melhorias nos processos da organização, porém, foram identificadas algumas mudanças negativas para os colaboradores e emergiram outros problemas. Buscando sanar essas dificuldades são apresentados insights baseados na literatura para que os gestores possam melhorar ou minimizar esses obstáculos apontados.

\section{Análise dos resultados}

Os resultados estão divididos em seis fatores chave ou categorias: planejamento estratégico, percepções dos colaboradores, treinamento dos colaboradores, consultoria externa, comprometimento da alta direção e comprometimento da área Tecnologia da Informação (TI).

Na categoria de planejamento estratégico é possível observar que todos os entrevistados foram informados com antecedência sobre a implantação do sistema e que ambos concordam que os fatores que contribuíram para a implantação do sistema foram os níveis de controle e a informação. Dumitru e Florescu (2009) reforçam a importância do planejamento estratégico justamente por direcionar os próximos passos e os objetivos da organização e, de quais formas a implantação do ERP pode contribuir para o alcance dos objetivos organizacionais.

Quanto às dificuldades existentes, os entrevistados destacaram que foi a adaptação das rotinas e tarefas advindas pelo sistema. $\mathrm{O}$ setor administrativo ainda complementa com outras dificuldades como resistência à mudança, qualidade das informações no sistema e falta de padrões de procedimentos. Em relação ao tema da resistência à mudança, ela pode ser natural do indivíduo (Nguyen et al., 2021; Jesus \& Oliveira, 2007; Macêdo et al., 2014; Souza \& Zwicker, 2000), sendo um fator relevante a considerar no processo de implantação, por isso estratégias podem ser adotadas visando minimizar a resistência.

Já sobre os fatores que contribuem para que a empresa não use plenamente o sistema ou da melhor forma possível, todos entrevistados destacam a infraestrutura, esse aspecto é visto como propulsor da implantação do ERP (Nguyen et al., 2021); o setor operacional destaca também a resistência, enquanto o setor administrativo complementa com fatores como experiência, treinamentos, cultura empresarial e qualidade das informações no sistema. Os fatores relacionados à cultura e capacidade dos colaboradores no manejo do sistema (Ferreira \& Kuniyoshi, 2015; Giorgia \& Santos, 2015) podem ser determinantes para o sucesso de implantação, portanto, definir metas e objetivos sobre o sistema também se faz de importante ocorrência (Souza \& Zwicker, 2000). 
$\mathrm{O}$ fator infraestrutura foi mencionado como motivo mais relevante para que o sistema não esteja sendo usado plenamente. Dessa forma, se o planejamento deve ser condizente com a estrutura organizacional existente (Nguyen et al., 2021; Ferreira \& Kuniyoshi, 2015; Giorgia \& Santos, 2015), pode-se afirmar, a partir das entrevistas, que apesar de ter ocorrido a informação sobre a ocorrência da implantação, não houve ou a empresa não teve condições de realizar avaliação da cultura presente e a capacidade dos colaboradores previamente ao processo de implantação, o que poderia resultar em utilização plena do sistema. Também não é possível avaliar se o sistema implantado foi moldado corretamente para as necessidades da empresa ou se houve necessidade da empresa se adaptar ao sistema, o que pode ter interferido no planejamento estratégico.

$\mathrm{Na}$ categoria percepções dos colaboradores, os integrantes entrevistados da área operacional relataram um baixo nível de controle antes da implantação do ERP, além de existir dificuldade ao acesso de informação pelo setor operacional; os dados eram lançados manualmente no sistema. O setor administrativo também relatou que não havia integração dos dados e não eram confiáveis. Após implantação ocorreu aumento dos níveis de controle, facilidade de acesso à informação, com aumento das áreas controladas, resultados similares aos estudos de Lindemayer, Soares e Mazon (2017) e Ferreira e Kuniyoshi (2015), em que houve alto percentual de integrantes reconhecendo o sucesso da implementação do sistema.

Já as mudanças negativas na percepção de todos os entrevistados são o aumento da demanda de trabalho, o setor operacional ainda destaca a eliminação de pessoas resistentes, ou seja, a dispensa de alguns colaboradores que se mantiveram resistentes às mudanças, enquanto que o setor administrativo dá ênfase no aumento da burocracia, falta de execução de todas as etapas dentro do sistema, tempo no processamento das informações e na tratativa das mesmas. As dificuldades em alterar procedimentos internos, insegurança dos usuários e possíveis boicotes ao sistema são fatores evidenciados no estudo de Gozzi et al. (2006), demonstrando que as organizações precisam estar preparadas para agir e evitar tais problemas.

Por fim, o grau de satisfação da implantação do sistema variou entre cinco e nove no âmbito geral, sendo pontuado pelo setor operacional entre oito e nove, e pelo setor administrativo entre cinco e nove. A justificativa da pontuação de todos os entrevistados foi devido ao fato que ainda existem pontos a serem melhorados e ao difícil entendimento das informações geradas pelo sistema, dizendo que o sistema deveria trazer as informações de forma mais visual ou ferramentas externas para visualização das informações. O setor operacional ainda destaca a falta de auto explicação das transações do sistema e que a estrutura 
do software não é auto explicativa, enquanto que o setor administrativo da ênfase que ainda existem procedimentos a serem melhorados e a falta de flexibilidade do software.

Desta forma, é de fácil compreensão que os colaboradores conhecem pontos que necessitam passar por melhorias e que é necessária uma análise das dificuldades entre os usuários do sistema quanto a análise das informações geradas pelo sistema, trazendo assim algum treinamento ou como comentado pelos próprios colaboradores, ferramentas para visualização de relatórios. Resultados similares foram observados no estudo de Almeida et al. (2016).

O setor operacional recebeu treinamento durante e após a implantação do sistema pelos usuários chave, enquanto que o administrativo recebeu antes e durante por consultoria externa, sendo responsáveis pela transmissão de conhecimentos aos colaboradores da área/setor operacional. O setor operacional destacou que as dúvidas e a base de dados para o treinamento não eram eficazes. O setor administrativo avaliou com nota cinco, classificando como básico, em uma visão geral do sistema, não transmitindo conhecimentos específicos. No âmbito operacional afirmam maior necessidade de treinamento objetivo, com foco em atividades a serem executadas e necessitam de atividade contínua, o administrativo descreve necessidade de objetividade nos recursos do ramo de atividade da empresa. A questão do treinamento é vista como um fator determinante para o bom funcionamento e para eficácia do uso do ERP (Dumitru \& Florescu, 2009).

Áreas operacional e administrativa afirmam que existe a necessidade de objetividade nas tarefas que serão executadas em seus treinamentos, indicando necessidade de melhoria neste aspecto. Dessa forma, Gozzi et al. (2006) apresentaram o treinamento como um fator importante no processo, além disso, o envolvimento do usuário e o apoio da direção. As respostas obtidas pelos usuários demonstram que faltou um planejamento adequado e definição clara das necessidades, significando necessidade de maior envolvimento da alta gestão. $\mathrm{O}$ treinamento é fator de sucesso, pois a adaptação inadequada dos usuários pode comprometer todo o projeto (Giorgia \& Santos, 2015).

O setor operacional teve acesso a um consultor para sanar dúvidas, enquanto o setor administrativo obteve acesso direto com os consultores externos especializados, que realizaram treinamentos, acompanhados de um consultor interno como gerente do projeto de implantação. A avaliação dos entrevistados foi diferente entre os setores: operacional avaliou como dez; administrativo avaliou entre cinco e oito; justificando que alguns consultores não eram qualificados o suficiente e a realização do pontapé inicial do projeto (GO live) aconteceu com muitas falhas. 
O fato de ter sido mencionado que o pontapé inicial de implantação ter ocorrido com muitas falhas, pode ser uma evidência de outros problemas, como: treinamento prévio com ênfase na entrada de dados e nas responsabilidades de cada operador pode não ter sido desenvolvido adequadamente (Jesus \& Oliveira, 2007; Oliveira \& Hatakeyama, 2012; Souza \& Zwicker, 2000); a resistência à mudança pode ter dificultado o processo de consultoria, uma vez que a resistência pode ser natural entre os envolvidos (Jesus \& Oliveira, 2007; Macêdo et al., 2014; Souza \& Zwicker, 2000), neste caso, uma maior proximidade com o setor administrativo pode ser o ponto de divergência entre o engajamento de um setor ou outro.

O setor operacional não teve contato direto com a alta administração no projeto de implantação do sistema, já o administrativo teve contato, mesmo assim, informam que houve pouco envolvimento da alta administração, o foco foi os prazos, falta de participação nas etapas do projeto, e o gerente de projeto não tinha conhecimento ou habilidades necessárias para o projeto.

A avaliação pelo setor operacional nas entrevistas foi de sete, considerando que a alta gestão disponibilizou recursos de infraestrutura necessários (com limitações), porém, sem contato direto, sem saber sobre o planejamento futuro. O setor administrativo avaliou entre seis e nove, informando falta de recurso financeiro para contratação de consultoria externa mais experiente, falta de envolvimento da alta administração no projeto e falta de recursos de infraestrutura. Faltou presença da alta gestão nas etapas e dificuldade de gestão pelo responsável pelo projeto. Gozzi et al. (2006) analisaram duas organizações, e aquela em que não houve envolvimento da alta gestão, atrelado aos outros fatores, teve diversos problemas em relação à adaptação do sistema as suas necessidades de negócio.

$\mathrm{Na}$ avaliação dos entrevistados a infraestrutura atende parcialmente ao sistema, no entanto, com elevado tempo de espera aos chamados, relatado pelo setor operacional com uma pontuação entre seis e dez, a infraestrutura atende adequadamente o fornecimento de periféricos, softwares e instalações. O setor administrativo pontua entre zero e dez, justificando que a equipe de TI é incompleta, prestação de serviço inadequado nos processos, serviço adequado no apoio técnico, mas não tem exclusividade para atividades no sistema.

A infraestrutura de TI, de um modo geral, atende parcialmente o sistema, com algumas indicações de falta de recursos de infraestrutura. Quanto aos processos, foi dita a palavra ineficaz, no sentido de que a equipe não presta serviços com exclusividade para o sistema ERP, ou pelo menos, não há equipe específica para tal. O estudo de Padilha e Marins (2005) apresentou a relevância da área de tecnologia da informação para o sucesso de implantação e pós implantação em uma organização, de forma que os usuários necessitam de aporte para 
cumprir com o objetivo do sistema. Dessa forma, o trabalho conjunto da infraestrutura é estratégico para que a fase de implementação e manutenção do funcionamento seja eficaz.

Dessa forma, os elementos favoráveis mencionados nas entrevistas após a implantação do ERP na organização foram as mudanças positivas observadas: aumento dos níveis de controle; fácil acesso à informação; aumento das áreas controladas; melhor gestão da documentação; registro de movimentações; integração dos dados; base única dos dados; melhora no clima organizacional; padronização dos processos; mudanças culturais pontuais; melhoria no fluxo dos processos; avaliação geral da implementação do sistema com média 7,0; avaliação geral dos treinamentos com média 6,8; avaliação geral da equipe de consultoria com média 8,1; avaliação geral do apoio da alta administração com média 7,1 e avaliação geral da equipe de TI com média 7,0.

Os aspectos positivos de aumento dos níveis de controle e integração dos dados são evidentes neste e em outros estudos (Ferreira, Penha, \& Kniess, 2019; Ferreira \& Kuniyoshi, 2015; Giorgia \& Santos, 2015; Gomes, 2013; Macêdo et al., 2014; Oliveira \& Hatakeyama, 2012; Roselli \& Cerqueira, 2016; Silvério et al., 2019; Souza \& Zwicker, 2003).

No Quadro 3 apresentam-se as mudanças negativas e relações dos problemas existentes observados pós implantação quanto ao uso do sistema, para atingir a utilização plena. Houve aumento de burocracia e demanda de trabalho na percepção dos entrevistados.

Quadro 3 - Relações das mudanças negativas e relações dos problemas existentes

\begin{tabular}{|c|c|}
\hline Relação das Mudanças Negativas & Relação dos Problemas Existentes \\
\hline $\begin{array}{l}\quad \text { Dificuldade Ainda Existente } \\
\checkmark \text { Adaptação; } \\
\checkmark \text { Qualidade das informações no sistema; } \\
\checkmark \text { Necessita de melhoria em alguns pontos; } \\
\checkmark \text { Falta de padrões de procedimentos. }\end{array}$ & $\begin{array}{l}\text { Aspectos Gerais } \\
\checkmark \text { Colaboradores com dificuldade na adaptação do } \\
\text { sistema; } \\
\checkmark \text { Resistência por parte dos colaboradores; } \\
\checkmark \text { Necessita de melhoria na infraestrutura; } \\
\checkmark \text { Falta de ferramentas para visualização de } \\
\quad \text { relatórios no sistema. }\end{array}$ \\
\hline $\begin{array}{l}\quad \text { Impeditivos de Uso Pleno do Sistema } \\
\checkmark \text { Melhorias na infraestrutura; } \\
\checkmark \text { Resistência por parte do colaborador; } \\
\checkmark \text { Falta de experiência; } \\
\checkmark \text { Falta de treinamentos; } \\
\checkmark \text { Cultura empresarial; } \\
\checkmark \text { Qualidade das informações imputadas no sistema. }\end{array}$ & \begin{tabular}{l}
\multicolumn{2}{c}{ Processos } \\
$\checkmark$ Qualidade das informações no sistema; \\
$\checkmark$ Falta de padrões de procedimentos; \\
$\checkmark$ Necessita de melhorias em alguns procedimentos.
\end{tabular} \\
\hline $\begin{array}{l}\quad \text { Aspectos Gerais } \\
\checkmark \text { Aumento da demanda de trabalho; } \\
\checkmark \text { Eliminação de pessoas resistentes; } \\
\checkmark \text { Aumento da burocracia; } \\
\checkmark \text { Falta de informação visual do software; } \\
\checkmark \text { Falta de flexibilidade do software. }\end{array}$ & $\begin{array}{l}\quad \text { Equipe de TI } \\
\checkmark \text { Tempo de espera elevado; } \\
\checkmark \text { Serviço inadequado quanto a processos; } \\
\checkmark \text { Falta de exclusividade de atividades voltadas ao } \\
\quad \text { sistema. }\end{array}$ \\
\hline
\end{tabular}

Fonte: Elaborado pelos autores. 
Observa-se necessidade de treinamentos e implementação de ferramentas de apoio na interpretação dos dados gerados pelo sistema. Os novos procedimentos que foram criados após a implantação precisam ser revisados, além da falta de qualidade das informações (alimentadas no sistema pelos próprios colaboradores), são pontos mencionados e que devem ser analisados pela organização. Algumas indicações realizadas por Macêdo et al. (2014) também se enquadram neste caso, como a necessidade de um sistema bem projetado, análise das fontes de resistência, com interfaces simples e fáceis de usar, são importantes para eliminar as dificuldades ainda existentes. Além disso, a teoria indica que a implantação do sistema força os profissionais a se aprimorarem (Peleias et al., 2009), o que resulta em resistência e em ações indesejadas pelas organizações, neste caso as dispensas de pessoas resistentes.

Por fim, as contribuições práticas gerenciais do estudo se dão das seguintes formas, a primeira, na qual foi possível levantar os pontos positivos e negativos da implantação do ERP sob a perspectiva dos colaboradores, que utilizam do ERP no dia a dia de suas atividades; informações pertinentes aos gestores da organização, a qual, principalmente, a partir dos pontos negativos os mesmos possam melhorar tais pontos. Na segunda forma, a partir dos achados dessa pesquisa, gestores que estejam planejando a implantação do sistema ERP podem se atentar às possíveis dificuldades que se dão no momento da implantação.

\section{Considerações finais}

Esta pesquisa teve como objetivo descrever as experiências dos colaboradores das áreas operacionais e estratégicas após o processo de implantação de um sistema ERP em uma indústria metalúrgica, atendendo a esse objetivo por meio de entrevistas semiestruturadas com seis colaboradores da organização.

A metodologia adotada foi suficiente para descobrir que, apesar das melhorias significativas no fluxo dos processos, níveis de controle, integração de dados, registros de movimentações, facilidade de acesso à informação e melhorias no clima organizacional, ainda existem dificuldades de adaptação ao novo sistema remanescente, falta qualidade nas informações e padrões nos procedimentos. Os colaboradores sugerem necessidade de treinamentos, para uma melhor experiência com o ERP. Essas conclusões, a partir da percepção dos colaboradores, também foram encontradas na literatura e sugerem que a empresa falhou em algumas fases cruciais do processo, principalmente no planejamento e envolvimento da alta direção na fase de implementação. 
Ao confrontar os resultados obtidos nesta pesquisa com aqueles apresentados por demais autores tomados como base para este estudo, como Ferreira, Penha e Kniess (2019), Ferreira e Kuniyoshi (2015), Grandón et al. (2018) Macêdo et al. (2014), Pinto et al. (2017) e Nguyen et al. (2021), nota-se a aderência dos resultados a uma realidade mais abrangente, vislumbrada em outras organizações dos mais variados ramos.

Isto fornece base interessante aos pesquisadores ao orientar futuras pesquisas para também se atentarem às potenciais dificuldades e fatores de sucesso da ferramenta, e também ao mercado sobre a importância de observar aspectos específicos da implantação, para que assim se potencialize o desempenho geral da ferramenta, maximizando ganhos e minimizando os problemas comumente enfrentados.

Há limitações do estudo no contexto da abrangência da pesquisa no que diz respeito à amostra pesquisada e à percepção dos respondentes no contexto e período pesquisado, que pode ainda ser complementada com outras análises, sobre a percepção de mais usuários internos e clientes externos. As entrevistas, apesar de serem realizadas com número reduzido de colaboradores, permitiram descrever a percepção e atingir o objetivo proposto. Essas limitações também permitem sugerir estudos futuros, com o mesmo método, em organizações de outros ramos de negócio e avaliação do sistema na perspectiva de outras categorias, como a percepção do cliente. $\mathrm{O}$ estudo também pode ser replicado e ampliado para múltiplos casos, objetivando comparar a implantação entre instituições.

\section{Referências}

Almeida, V. E., Kudlawicz-Franco, C., \& Sokulski, C. C. (2016). Implantação de ERP: estudo de caso sobre a resistência à mudança organizacional. Revista Organização Sistêmica, 8(5), 40-58.

Appolinário, F. (2011). Dicionário de metodologia científica: um guia para a produção do conhecimento científico (2.ed.). São Paulo: Atlas.

Bancroft, N. H., Seip, H., \& Sprengel, A. (1998). Implementing SAP R/3: how to introduce a large system into a large organization (2.ed.). Greewich: Manning.

Bardin, L. (2011). Análise de conteúdo. São Paulo: Edições 70.

Bernardes, E., Muniz Junior, J., \& Nakano, D. (2019). Pesquisa qualitativa em engenharia de produção e gestão de operações. São Paulo: Atlas.

Caiçara Junior, C. (2012). Sistema integrados de gestão-ERP: uma abordagem gerencial. Curitiba/PR: InterSaberes. 
Colangelo Filho, L. (2001). Implantação de sistema ERP (Enterprise Resource Planning): um enfoque de longo prazo. São Paulo: Atlas.

Cooper, D. R., \& Schindler, P. S. (2016). Métodos de pesquisa em administração (12.ed.). Porto Alegre: AMGH.

Correa, J., \& Spinola, M. de M. (2015). Adoção, seleção e implantação de um ERP livre. Production, 25(4), 956-970.

Costa, C. J. et al. (2016). Enterprise resource planning adoption and satisfaction determinant. Computers in Human Behavior, 63, 659-671.

Dumitru, V., \& Florescu, V. (2009). Enterprise resource planning deployment guide. Amfiteatru Economic, 11(25), 213-222.

Esteves, J. M., Carvalho, J. A., \& Santos, A. A. (2000). O ciclo de vida dos custos dos sistemas ERP. Anais do VII Congresso Brasileiro de Custos, Recife, PE, Brasil, 7.

Ferreira, A. A., \& Kuniyoshi, M. S. (2015). Critical factors in the implementation process of integrated management systems. Journal of Information Systems and Technology Management, 12(1), 145-164.

Ferreira, W. S., Penha, R., \& Kniess, C. T. (2019). Contribuições de um software ERP para escritórios de arquitetura. IPTEC - Revista Inovação, Projetos e Tecnologias, 7(2), 142-155.

Giorgia, M., \& Santos, W. S. (2015). Sistema ERP: um estudo exploratório numa empresa do setor têxtil do agreste pernambucano. Revista Brasileira de Gestão e Inovação - Brazilian Journal of Management \& Innovation, 2(3), 64-76.

Gomes, R. M. S. (2013). Contributions of the PMBOK to the Project management of an ERP system implementation. Revista de Gestão de Projetos - GeP, (2), 153-162.

Gozzi, S., Fedichina, M. A. H., Olivo, R. L. F., \& Machado, M. C. (2006). Competitividade organizacional por meio do sistema ERP: uma análise dos resultados alcançados na fase da pós-implantação. Revista Organizações em Contexto, 2(4), 9-27.

Grandón, E. E., Ramírez-Correa, P. E., \& Rojas, K. P. (2018). Uso de la teoría business process change (BPC) para examinar la adopción de enterprise resource planning (ERP) em Chile. Interciencia, 43(10), 716-722.

Groenewald, D., \& Okanga, B. (2019). Optimising enterprise resource planning system to leverage a firm's absorptive and adaptive capabilities. South African Journal of Information Management, 21(1), 1-15.

Jesus, R. G., \& Oliveira, M. O. F. (2007). Implantação de sistemas ERP: tecnologia e pessoas na implantação do SAP R/3. Journal of Information Systems and Technology Management, 3(3), 315-329.

Lindemayer, E., Soares, T. C., \& Mazon, G. (2017). Implantação do Sistema de Gestão - Um Estudo de Caso. IPTEC - Revista Inovação, Projetos e Tecnologias, 5(1), 124-136. 
Macêdo, D. G., Gaete, L., \& Joia, L. A. (2014). Antecedentes à Resistência a Sistemas Empresariais: A Perspectiva de Gestores Brasileiros. RAC, Rio de Janeiro, 18(2), 139-160.

Nguyen, X. H. et al. (2021). Determinants of Implementation of Enterprise Resource Planning System: A Case Study in Vietnam. Journal Of Asian Finance Economics And Business, 8(4), 501-506.

Oliveira, L. S. de, \& Hatakeyama, K. (2012). Um estudo sobre a implantação de sistemas ERP: pesquisa realizada em grandes empresas industriais. Produção, 22(3)596-611.

Padilha, T. C. C., \& Marins, F. A. S. (2005). Sistemas ERP: características, custos e tendências. Produção, 15(1), 102-113.

Pavón González, Y., Puente Baró, L., Infante Abreu, M., \& Blanco González, J. (2018). Experiencia de trabajo para la configuración del ERP Odoo en pequeños negocios. Caso de éxito en TostoneT. Ingeniare. Revista chilena de ingeniería, 26(3), 514-527.

Peleias, I. R., Trevizoli, J. C., Cortes, P. L., \& Galegale, N. V. (2009). Pesquisa sobre a percepção dos usuários dos módulos contábil e fiscal de um sistema ERP para o setor de transporte rodoviário de cargas e passageiros. Revista de Gestão da Tecnologia e Sistemas de Informação, 6(2), 247-270.

Pinto, S. M., Ramírez, P. E., \& Grandón, E. E. (2017). Antecedentes del Éxito de los Sistemas de Planificación de Recursos Empresariales en las Grandes Empresas Chilenas: un Modelo Factorial Exploratorio. Información Tecnológica, 28(3), 139-146.

Roselli, K., \& Cerqueira, L. S. (2016). Avaliação do impacto da implementação do sistema ERP em uma empresa de comércio exterior na cidade de Rio Grande-RS. Revista de Tecnologia Aplicada (RTA), 5(3), 27-45.

Sammon, D., \& Adam, F. (2010). Project preparedness and the emergence of implementation problemas in ERP projects. Information \& Management, 47(1), 1-8.

Silvério, A. O., Santos, L. A., Bastos, C. E. (2019). A importância da utilização de um sistema ERP para o gerenciamento de estoque. Anais do VI Congress of Industrial Management and Aeronautical Technology, São José dos Campos, SP, Brasil, 6.

Souza, C. A. de, \& Zwicker, R. (2003). Big-bang, small-bangs ou fases: estudo dos aspectos relacionados ao modo de início de operação de sistemas ERP. Revista De Administração Contemporânea, 7(4), 9-31.

Souza, C. A. de, \& Zwicker, R. (2000). Ciclo de vida de sistemas ERP. Caderno de Pesquisas em Administração, 1(11), 46-57.

Yin, R. (2018). Case study research and applications: design and methods (6.ed.). Los Angeles: SAGE. 